\title{
Uso de Redes Sociales en el Proceso de Enseñanza y Aprendizaje por los Estudiantes y Profesores de la Universidad Antonio Nariño, Sede Cartagena
}

\author{
Lorcy P. De La Hoz ${ }^{(1) \star}$, Diofanor Acevedo(2) y Juan Torres ${ }^{(3)}$ \\ (1) Universidad Antonio Nariño, Facultad de Comercio Internacional, Avenida Crisanto Luque, Dg. 22, \\ No. 48 A - 64. Cartagena, Bolívar-Colombia (e-mail: lorcydelahoz@uan.edu.co) \\ (2) Universidad de Cartagena, Facultad de Ingeniería, Programa de Ingeniería de Alimentos, Avenida el \\ Consulado, Calle 30, No. 48-152. Cartagena, Bolívar-Colombia \\ (3) Universidad Antonio Nariño, Facultad de Ingeniería Electrónica y Biomédica, Programa de Ingeniería \\ Electrónica, Avenida Crisanto Luque, Dg. 22, No. 48 A - 64. Cartagena, Bolívar-Colombia \\ * autor a quien debe ser dirigida la correspondencia
}

Recibido Dic. 22, 2014; Aceptado Mar. 5, 2015; Versión final recibida Mar. 16, 2015

\begin{abstract}
Resumen
Las redes sociales son plataformas en la Internet que facilitan el intercambio de información, la interacción y la colaboración entre sus usuarios. En el presente estudio se evaluó el uso de las redes sociales en el proceso de enseñanza y aprendizaje por los estudiantes y profesores de la Universidad Antonio Nariño, sede Cartagena. Se utilizó un diseño de tipo no experimental descriptivo. Se realizó una encuesta estructurada, con 20 preguntas y un total 29 ítems a estudiantes y profesores. Casi $32 \%$ del total de encuestados utiliza las redes sociales como herramienta educativa, quedando solo por debajo del chateo entre familiares y amigos. El estudio demuestra que el uso de las redes sociales por estudiantes y profesores de la Universidad Antonio Nariño en los procesos pedagógicos es aceptable, pero puede incrementarse creando una política institucional en el manejo y aplicación de las nuevas tecnologías de la informática y la comunicación en general.
\end{abstract}

Palabras clave: redes sociales, enseñanza, aprendizaje, conectivismo

\section{Using Social Networks in the Process of Teaching and Learning by Students and Teachers of the University Antonio Nariño, Campus Cartagena}

\begin{abstract}
Social networks are platforms on the Internetthat facilitate information sharing, interaction and collaboration among its users. In this study the use of social networks in the process of teaching and learning by the students and faculty of the University Antonio Nariño, Campus Cartagena, was evaluated. A not experimental descriptive design was used. A structured survey, with 20 questions and a total 29 items to students and teachers. Close to $32 \%$ of respondents used social media as an educational tool, being just below the chat between friends and family. The study shows that the use of social networks by students and professors of the University Antonio Nariño, campus Cartagena, in learning processes is acceptable, but can be increased by creating an institutional policy on the management and implementation of new information and communication technologies.
\end{abstract}

Keywords: social networking, teaching, learning, classroom, distance, connectivism 


\section{INTRODUCCIÓN}

Las redes sociales son estructuras formadas por individuos vinculados por motivos como la amistad, parentesco, ideas, aficiones, relaciones de trabajo, entre otras. Las redes sociales constituyen un sistema abierto de construcción permanente, que involucra a personas que se identifican con necesidades y problemas similares, cuyos principios son el de crear, compartir y colaborar (Vidal et al., 2013). El primer uso de redes sociales en el ámbito académico aparece con el uso de las herramientas de comunicación interpersonal y colaboración en red en el contexto educativo. En este sentido, son numerosas las posibilidades que brindan las redes sociales en la práctica docente (Vidal et al., 2013). Actualmente la Web 2.0 y las herramientas informáticas están adquiriendo cada día más protagonismo en la sociedad. Como parte de la misma, la trascendencia de estas herramientas en la docencia es cada vez mayor (Wodzicki et al., 2012). Por ello, son muchos los trabajos que se pueden encontrar en los que se propone la incorporación de la Web 2.0 en actividades docentes, con el objetivo de favorecer nuevos modelos de enseñanza y aprendizaje (Falahah y Rosmala, 2012; Roblye et al., 2010).

Las redes sociales abren múltiples opciones para añadir una nueva dimensión a los procesos de aprendizaje y conocimiento. En particular, los sitios de redes sociales permiten a los estudiantes conectarse a entornos de aprendizaje formales e informales, que puedan encontrar personas de ideas afines y organizar el intercambio de conocimiento informal con fines educativos (Wodzicki et al., 2012). Estas características hacen pensar que su uso sería conveniente en entornos educativos con el fin de potenciar diversos aspectos como la participación del estudiantado en el proceso de enseñanza y aprendizaje, aprendizaje autónomo, interacción y motivación de los estudiantes, creatividad del estudiante y la creación de redes de colaboración e intercambio con continuidad. Sin embargo, el uso de redes sociales en la docencia universitaria, plantea diversas cuestiones con respecto a si favorece o no el uso de estas en el trabajo educativo (Mendiguren et al., 2012).

En los últimos años, el uso de las tecnologías de información en la docencia está en auge (Kimmons y Veletsianos, 2014), y los medios de comunicación social ofrecen al estudiante la oportunidad de manipular su entorno de aprendizaje y de participar activamente en el proceso de aprendizaje, esto podría permitir a los diseños centrados en el estudiante y el aprendizaje autodirigido (Wodzicki et al., 2012). Promover el aprendizaje autodirigido es uno de los retos más importantes de los sistemas educativos de hoy en día, porque la adaptación a entornos que cambian rápidamente se está convirtiendo cada vez más importante (Schmitz et al., 2007). Es un reto para promover el aprendizaje autodirigido de los estudiantes con los medios de comunicación social en la educación formal (Väljataga y Fiedler, 2009). Por otra parte, los medios sociales permite una creación colectiva del conocimiento, un proceso en el que no sólo el miembro va a aprender mucho, pero también un pedazo de conocimiento colectivo evolucionará (Moskaliuk et al., 2009).

Los adolescentes y adultos jóvenes usan los medios de comunicación en alto grado (Wodzicki et al., 2012). De acuerdo con Wodzicki et al., (2012), el $88,3 \%$ de los estudiantes universitarios son propietarios de sus propias computadoras portátiles en el 2009 en comparación con 65,9\% en 2006, y gastó un promedio de 21,3 horas a la semana en línea. Algunas voces en el debate actual, incluso han exigido cambiar la educación, con el fin de responder adecuadamente a la utilización frecuente de los medios de comunicación social de los jóvenes (Wodzicki et al., 2012). Los medios sociales proporcionan múltiples oportunidades que pueden ser aprovechadas por los procesos de aprendizaje y conocimiento en general. Esto conduce a un aumento del uso de los medios sociales en los entornos de aprendizaje formal, por ejemplo, el uso de sitios de redes sociales para el aprendizaje del idioma Inglés (Kabilan et al., 2010), como un repositorio de información para un curso mixto (Arnold y Paulus, 2010) o como una herramienta para desarrollar la identificación de los estudiantes con un grupo de aprendizaje y la tarea de aprendizaje (Augustsson, 2010).

Las redes sociales se han venido implementando en los diferentes niveles educativos desde tres perspectivas: como complemento a los cursos presenciales, como entorno principal para la enseñanza o como un foro para la comunicación de conocimientos en red (Falahaha y Rosmala, 2012; Kabilan et al., 2010; Arnold y Paulus, 2010). En Colombia han sido muchas las experiencias que han mostrado las bondades de la utilización de la la Web 2.0 y las Tecnologías de la Información y la comunicación (TIC) en el proceso de enseñanza y aprendizaje por parte de los estudiantes y profesores y sus procesos de pensamiento. Sin embargo, se han hecho limitadas investigación sobre estos aspectos (Molina y Briseño, 2005). Teniendo en cuenta lo anterior, el objetivo de esta investigación fue determinar el uso de las redes sociales en el proceso de enseñanza y aprendizaje por los estudiantes y profesores de la Universidad Antonio Nariño (UAN) sede Cartagena. Estudios de este tipo son escasos en Colombia y en particular la Costa Caribe. 


\section{MATERIALES Y MÉTODOS}

\section{Diseño de la investigación}

Se abordó un diseño de tipo no experimental descriptivo. Para la recogida de los datos se aplicó, como único instrumento de recolección de información una encuesta estructurada similar al propuesto y validado por otros autores (Flores y López, 2014; Cabero y Marín, 2013; Falahah. y Rosmala, 2012). El cuestionario consistía en 20 preguntas y un total 29 ítems a estudiantes y profesores con el fin de obtener la información referente a los objetivos planteados. La encuesta tuvo dos tipos de preguntas cerradas donde se le daba la opción a los encuestados de elegir si, no y otras de opción múltiple. Se aplicó de manera virtual a través de la herramienta de Google drive y fue la misma para estudiantes y profesores. Se tituló "Influencia de las Redes Sociales en la Universidad Antonio Nariño".

\section{Población y muestra}

La población con la cual se trabajó fue de 348 unidades, de las cuales 310 son estudiantes y 38 profesores de todos los programas existentes en la UAN de la ciudad de Cartagena. Por medio de un listado proporcionado por los directores de programa se visitaron las aulas de clase, se enviaron correos electrónicos a los estudiantes y se los solicitó responder voluntaria y anónimamente la encuesta presentada. De manera similar se les hizo la solicitud a los profesores. Para calcular la muestra representativa de la población 348 unidades, se utilizó una fórmula estadística con la cual se obtuvieron 176 unidades muéstrales. Distribuidas de la siguiente manera: 20 corresponden a los profesores y 156 a los estudiantes. Se utilizó el procedimiento por estrato (López, 2004) que consiste en subdividir a la población en subgrupos homogéneos, se establecieron proporciones y se llegó al resultado de la Tabla 1.

Tabla 1: Procedimiento por estrato para escoger muestra

\begin{tabular}{|c|c|c|c|}
\hline \multicolumn{2}{|c|}{ Población } & \multicolumn{2}{c|}{ Muestra } \\
\hline Estudiantes & Profesores & Estudiantes & Profesores \\
\hline 310 & 38 & 156 & 20 \\
\hline 348 & \multicolumn{2}{c|}{176} \\
\hline
\end{tabular}

\section{Planificación y aplicación del trabajo de campo}

Para el desarrollo de la presente investigación se tuvieron en cuenta cuatro etapas.

Etapa I: en esta etapa se determina la presentación del proyecto, una vez aprobado se procede a realizar un levantamiento del estado del arte de la fundamentación teórica del uso de las redes sociales en los procesos de enseñanza y aprendizaje, tras la recopilación de fuentes y revisión documental se procede al análisis, clasificación de la información que permitan determinar los referentes teóricos aplicables a la misma. Está etapa de la investigación se empezó a desarrollar a partir del mes de abril del 2013 hasta el mes de octubre del mismo año, y fue a la par de las otras etapas en aras de tener la documentación más actual posible que exista sobre el tema que se lleva a cabo.

Etapa II: está caracterizada por la definición del diseño de la investigación: desarrollo metodológico a partir de los paradigmas de investigación cuantitativa y cualitativa, enfocado en un sondeo de tipo descriptivo y la definición de la encuesta y la observación como técnicas para la recolección de la información que busquen dar respuesta a los objetivos planteados.

Etapa III: Se diseña la encuesta, una vez se tiene definido la población y muestra y establecido el cuestionario se procede a su aplicación, para efectos de la investigación se opta por realizar un cuestionario cerrado que se resolverá de manera virtual previa autorización de las autoridades académicas y aprobación de los estudiantes y profesores seleccionados al azar para su posterior envió, la encuesta se envía a los correos electrónicos con una respectiva carta solicitando el desarrollo del mismo y su agradecimiento por participar en la investigación. Consecutivamente se va dando respuesta a los cuestionarios se tienen las estadísticas de los mismos lo que facilita su posterior análisis e interpretación. El tiempo aproximado en el diligenciamiento del cuestionario fue de 3 a 5 minutos, y la aplicación del número total de encuestas 176 duró aproximadamente dos meses, finalizando en el mes de noviembre de 2013 que coincide con la entrega del segundo informe de seguimiento.

Etapa IV: Una vez se ha realizado el análisis e interpretación de los datos se procede a la redacción y presentación de la síntesis y las conclusiones de los mismos. 


\section{RESULTADOS Y ANÁLISIS}

\section{Población y muestra}

La muestra intencional se aplicó de acuerdo al número de estudiantes inscritos por programa de los cuales un $22,4 \%$ son de ingeniería electromecánica, $20,5 \%$ de electrónica, seguidos con un $17,9 \%$ de Comercio Internacional y el resto distribuido de la siguiente manera: 7,7\% de ingeniería industrial y administración de empresas, 7,1\% corresponden a tecnología en electromecánica, 6,4\% contaduría y 5,1\% ingeniería biomédica y psicología. El perfil general de los encuestados muestra una población altamente masculina con un $60,2 \%(n=106)$, de los cuales $88,6 \%$ son estudiantes y $11,4 \%$ profesores, frente a un $39,8 \%$ de mujeres $(n=70)$, donde $36,4 \%$ son profesoras y $63,6 \%$ estudiantes. En cuanto al nivel de formación se puede observar que los profesores en un 5,7\% $(n=10)$ tienen estudios de postgrado: 2,8\% especialización $(n=5), 2,3 \%$ maestría $(n=4)$ y $0,6 \%$ con doctorado $(n=1)$, lo cual evidencia que el mayor número de profesores en la universidad son especialistas y hay un número insignificante de doctores. Este hecho también permite visualizar que los profesores tienen un interés por mantenerse actualizados. Cabe resaltar que la población la conforman inmigrantes digitales dado a las edades encontradas.

Términos como "Generación Net" o nativos digitales (Wodzicki et al., 2012) se utilizan a menudo para etiquetar la generación nacida después de 1980, aquellas personas que se han criado con estas innovaciones como parte inherente de su vida (Bennett et al., 2008). En la Tabla 2 se puede observar que una parte de la muestra analizada en esta investigación es adulta, y que no alcanzaron a ser nativos digitales y que en cierta forma la incursión en la Web 2.0 para muchos es un desafío (Cabra-Torres y MarCiales-ViVas, 2009). Algunos investigadores (Wodzicki et al., 2012; Bennett et al., 2008) argumentan que los nativos digitales poseen, como resultado de su exposición temprana a estas tecnologías, las habilidades más desarrolladas relativas a las solicitudes de Internet y, aún más allá de eso, que aprendan de una manera que es fundamentalmente diferente de las generaciones anteriores. Un argumento inherente, pero nunca probado hasta ahora, es la suposición de que la Generación Net maneja las redes sociales de forma más eficaz y eficiente, tanto para fines sociales como de aprendizaje.

Tabla 2: Rango de edades de la muestra estudiada $(n=176)$

\begin{tabular}{|c|c|c|c|c|}
\hline \multirow{2}{*}{ Edad } & \multicolumn{2}{|c|}{ Estudiantes } & \multicolumn{2}{c|}{ Profesores } \\
\cline { 2 - 5 } & $\mathrm{N}$ & $\%$ & $\mathrm{~N}$ & $\%$ \\
\hline $20-25$ & 63 & $35,8 \%$ & 1 & $0,6 \%$ \\
\hline $26-30$ & 48 & $27,3 \%$ & 4 & $2,3 \%$ \\
\hline $31-35$ & 28 & $15,90 \%$ & 2 & $1,10 \%$ \\
\hline $36-40$ & 14 & $8,0 \%$ & 2 & $1,10 \%$ \\
\hline más de 41 & 3 & $1,7 \%$ & 11 & $6,30 \%$ \\
\hline Total & 156 & $88,7 \%$ & 20 & $11,3 \%$ \\
\hline
\end{tabular}

Del total de encuestados el $94,9 \%$ ( $n=167)$ pertenece a alguna red social, de los cuales $85,2 \%(n=150)$ son estudiantes. Los motivo por los cuales el $5,1 \%$ restante $(n=9)$ no hacen parte de las redes sociales son: con un $2,3 \%(n=4)$ no están bien informados sobre ellas, el $1,7 \%(n=3)$ dice no tener tiempo y prefieren ocupar su tiempo libre en otras actividades y el $1,1 \%(n=2)$ no les interesa. Se pudo observar que ninguno manifestó tener dificultades de acceso a internet. Si bien es cierto que la implementación de las redes sociales en el aula por sí solas no es garantía para mejorar el nivel académico, la percepción de que estas mejoran el nivel académico en los encuestados fue de $70,5 \%$ para estudiantes y $60 \%$ para profesores. Con respecto al grado de incidencia en el nivel académico se encontró que el $87,1 \%$ de los estudiantes encuestados afirman que las redes sociales tienen influencia en el mejoramiento de los niveles académicos y que están de acuerdo en que estas se utilicen en el aula como herramientas pedagógicas, y afirman que pueden ser usadas como herramientas académicas. En cuanto a los profesores el $80,0 \%$ también lo estuvieron.

Estos resultados concuerdan con los de Morduchowicz (2012), quien plantea que la popularidad con que cuentan las redes sociales entre los jóvenes universitarios conlleva a que estas puedan ser utilizadas como recursos colaborativos donde interactúan profesores y estudiantes. Falahah y Rosmala (2012) en su investigación del uso de las redes sociales en el entorno de la educación superior, especialmente entre profesores y estudiantes, y el análisis del impacto en la actividad de enseñanza-aprendizaje de 300 encuestados en tres universidades privadas familiarizadas con las actividades de redes sociales encontraron que la mayoría de los encuestados están de acuerdo para el libre acceso a las redes sociales en horario de oficina, y en cerca de $60 \%$ de los encuestados utilizan éstas no sólo para entretenerse, sino también para la distribución de la información y la comunicación para apoyar la actividad docente. Los usos 
varían desde asignación de tareas, anuncios, negociaciones de clase de reprogramación, exámenes, y así sucesivamente, utilizando aplicaciones como Facebook, Twitter, mensajería instantánea y el sitio de blog.

Según la Tabla 3, la red social más usada por estudiantes y profesores es Facebook. Si bien es cierto que el objetivo inicial con que fue creada esta red no fue el académico, su altísima conectividad externa, y la flexibilidad en la gestión de tareas la convierten en la mejor opción para desarrollar actividades académicas colaborativas. Facebook quizás sea hoy el ejemplo más palpable de los entornos denominados redes sociales. Afirman que las redes sociales son plataformas que facilitan el intercambio de información, la interacción y la colaboración entre sus usuarios. Para estos autores el éxito de Facebook como red social, sin embargo, no depende sólo de su capacidad para conectar personas, aunque sea esta su orientación inicial, y la potencia de la plataforma para compartir recursos, para vincular contenidos presentes en internet a los perfiles de los usuarios y su evolución hacia el lifestreaming y el microblogging la facultan para dar soporte a experiencias de interacción complejas y continuas, y, con ello, para estructurar procesos de aprendizaje colaborativo. Las herramientas comunicativas de la plataforma, así como la opción de enriquecer sus potencialidades mediante la instalación de aplicaciones y módulos de terceros permiten a los miembros de una comunidad educativa o equipo de trabajo desarrollar actividades heterogéneas (Llorens \& Capdaferro, 2011)).

Tabla 3: Redes Sociales más usadas $(n=167)$

\begin{tabular}{|c|c|c|c|c|c|c|c|c|}
\hline & Facebook & Twitter & Lindklin & Google+ & Eduteka & Educared & Badoo & Total \\
\hline Profesores & 5,0 & 3,0 & 2,0 & 4,0 & 1,0 & 2,0 & 0,0 & 17 \\
\hline$\%$ & 29,4 & 17,6 & 11,8 & 23,5 & 5,9 & 11,8 & 0,0 & 100 \\
\hline Estudiantes & 70,0 & 15,0 & 4,0 & 55,0 & 3,0 & 3,0 & 6,0 & 150 \\
\hline$\%$ & 46,7 & 10,0 & 2,7 & 36,7 & 2,0 & 2,0 & 4,0 & 100 \\
\hline
\end{tabular}

Facebook es uno de los ejemplos más recientes de que las tecnologías de comunicación han sido adoptados ampliamente por los estudiantes y, en consecuencia, tienen el potencial de convertirse en un recurso valioso para apoyar sus comunicaciones educativas y colaboraciones con el profesorado (Roblye et al., 2010). Facebook proporciona un espacio virtual en el que un grupo de trabajo puede discutir, opinar, organizar acontecimientos, enviar información, compartir ideas, propuestas y elaborar contenidos, creando así lo que se denomina una comunidad virtual de aprendizaje (Llorens \& Capdaferro, 2011). Cuando el motivo principal de la existencia de una comunidad pasa de ser el solo intercambio de información a ser el de aprendizaje y desarrollo profesional, entonces nos encontramos ante una comunidad virtual de aprendizaje (Llorens \& Capdaferro, 2011; McMillan y Chavis, 1986).

En la Tabla 4 se observa que $31,74 \%(n=53)$ del total de encuestados utiliza las redes sociales como herramienta educativa, quedando solo por debajo del chateo entre familiares y amigos $(32,34 \%)$, lo cual evidencia que las redes sociales tienen gran importancia en la vida educativa de los estudiantes de la UAN sede Cartagena. Estas redes tecnológicas de comunicación se han convertido en una herramienta que permite el aprendizaje colaborativo e involucra espacios de intercambio de información que fomentan la cooperación (Islas y Carranza, 2011). Este estudio muestra que no deben ser obviadas, ya que su arraigo y fascinación en los estudiantes son una posibilidad didáctica enorme; una muestra de ellos que es que su uso en las universidades América Latina, es cada vez más notorio (Islas y Carranza, 2011).

Tabla 4: Mayor uso de redes sociales por encuestados $(n=167)$

\begin{tabular}{|l|c|c|}
\hline \multicolumn{1}{|c|}{ Uso de la red social } & Frecuencia & $\%$ \\
\hline Enviar y recibir mensajes a familiares y amigos & 54 & 32,34 \\
\hline Intercambiar información y conocimiento & 29 & 17,37 \\
\hline Motivo de estudio o profesional & 24 & 14,37 \\
\hline Intercambiar multimedia & 21 & 12,57 \\
\hline Participar en debates & 21 & 12,57 \\
\hline Diversión y ocio & 18 & 10,78 \\
\hline Total & 167 & 100 \\
\hline
\end{tabular}

En la Tabla 5 se evidencian las horas al día que dedican los encuestados a las redes sociales en el ámbito educativo. Esta evidencia que por lo menos el $86,8 \%(n=46)$ de los encuestados dedica hasta cuatro horas a las redes sociales, lo cual demuestra el potencial uso de las redes sociales en los procesos pedagógicos para comentar tareas, enviarse material, buscar información, visitar perfiles de empresas, entre otras. La utilización de redes sociales, constituyen nuevas muestras de la vigencia y procedencia de los principios 
que sustentan la educación para todos, el aprender a aprender, la enseñanza y aprendizaje personalizada, la imprescindible educación para toda la vida que, en definitiva, contribuyen a materializar, de manera concreta y efectiva, la educación permanente o continua, haciendo uso de la educación a distancia, la cual, reduce con eficacia, los obstáculos que representan el tiempo y el espacio; en ella, se recurre a métodos, técnicas y recursos que elevan la productividad y la flexibilidad del proceso de enseñanza y aprendizaje (Alfonso, 2003). A la pregunta que si estaría dispuesto a recibir capacitación para trabajar en las redes sociales el $94 \%$ estudiantes respondió afirmativamente mientras, por su parte los profesores en un $100 \%$ están dispuestos a recibir capacitación para la implementación de las redes sociales como parte del proceso de enseñanza y aprendizaje.

Tabla 5: Horas al día dedicas a redes sociales en el ámbito educativo $(n=53)$

\begin{tabular}{|c|c|c|}
\hline Horas dedicadas & Frecuencia & Porcentaje \\
\hline 1-2 h al día & 29 & 54,72 \\
\hline 3-4 h al día & 14 & 26,42 \\
\hline $4-5$ h al día & 3 & 5,66 \\
\hline 5-6 h al día & 3 & 5,66 \\
\hline 6 en adelante & 4 & 7,55 \\
\hline Total & 53 & 100 \\
\hline
\end{tabular}

El $95,5 \%$ de los estudiantes y el $95 \%$ de los profesores, le gustaría que la universidad contara con su propia red social de aprendizaje. Según Llorens \& Capdaferro (2011) las redes sociales favorecen la cultura de comunidad virtual y el aprendizaje social. Desde un punto de vista psicosociológico, esta cultura se fundamenta en valores que surgen de los usuarios, que interactúan en la red en torno a un tema u objetivo común y que generan lazos interpersonales de confianza, apoyo, sentimiento de pertenencia e identidad social. Por otro lado, la existencia de redes de intercambio y flujos de información es un aspecto relevante en la configuración y mantenimiento de una red social.

De los resultados se evidencian potenciales usos de las redes sociales para soportar experiencias de aprendizaje colaborativo. Estas hacen parte activa de la vida de las comunidades, por lo que es necesario implementar enfoques colaborativos en los modelos pedagógicos que contribuyan en la mejora de los niveles académicos. Modelos pedagógicos recientes como el conectivismo tienden a derribar las rígidas fronteras entre el aprendizaje formal e informal. Este modelo se fundamenta en las limitaciones de las teorías conductista, cognitivista y constructivista frente a los efectos de la tecnología sobre la forma en que actualmente vivimos, nos comunicamos y aprendemos. Esta teoría nace debido al advenimiento de las denominadas Tecnologías de la Información y las Comunicaciones (TICs) y su incidencia en la manera como el hombre del siglo XXI accede al conocimiento y desarrolla nuevas formas de aprendizaje (Moreno, 2011). A consecuencia del potencial manifiesto de las nuevas tecnologías y redes sociales como herramientas para uso del saber colectivo, estas presentan posibilidades pedagógicas extraordinarias, en particular si se está dispuesto a mirar con nuevos ojos los conceptos de formación y capacitación, y si se pone el énfasis en el carácter social de la construcción del conocimiento (Llorens \& Capdaferro, 2011).

Con los resultados obtenidos en la presente investigación podemos evidenciar que las redes sociales se están convirtiendo en una herramienta que permite el desarrollo de las habilidades comunicativas y que podría ser útil en los procesos de enseñanza y aprendizaje puesto en práctica por docente y estudiante en su quehacer académico, teniendo en cuenta que la mayoría de estos usan estas herramientas un tiempo considerable, aun cuando estos consideren que lo hacen sin ningún beneficio específico, a pesar de que sí las incluyen como parte de sus actividades académicas. Teniendo en cuenta lo anterior, las redes tiene gran potencial para hacer una transformación educativa que permitiría espacios de diálogo propicios para el aprendizaje y enriquecimiento mutuo entre docentes y estudiantes; es decir, un sitio donde la interacción, individualidad y multiculturalidad se respeten, y, al mismo tiempo, enriquezcan el aprendizaje colaborativo y fomenten la metacognición de los actores con actividades que conlleven a la autoevaluación o reflexión sobre el trabajo realizado (Islas y Carranza, 2011).

\section{CONCLUSIONES}

El estudio demuestra que el uso de las redes sociales por estudiantes y profesores de la Universidad Antonio Nariño sede Cartagena en los procesos pedagógicos es aceptable, pero puede incrementarse creando una política institucional en el manejo y aplicación de las nuevas tecnologías de la informática y la comunicación en general. La red social más usada por estudiantes y profesores es Facebook, seguida de Google+. La experiencia de las redes sociales con fines académicos que han tenido los estudiantes de la 
UAN sede Cartagena ha partido de iniciativas individuales de algunos profesores. No obstante, la institución presenta una disponibilidad para la implementación de las redes sociales aunque no cuenta con la concreción de un proyecto para la capacitación de profesores y estudiantes. Estas deficiencias en el buen uso de las redes sociales se deben a la falta de estrategias institucionales en torno a la implementación de estas, ya que a la fecha hay pocas reflexiones en torno a cómo relacionar el uso de las redes con los objetivos de la Universidad en las áreas de enseñanza, investigación, administración y el cómo usar redes sociales para incrementar el capital relacional, inteligencia e identidad.

\section{REFERENCIAS}

Alfonso, I.R., La educación a distancia, ACIMED, 11(1), 3-4 (2003).

Arnold, N. y T. Paulus, Using a social networking site for experiential learning: Appropriating, lurking, modeling and community building, The Internet and Higher Education, 13(4), 188-196 (2010).

Augustsson, G., Web 2.0, pedagogical support for reflexive and emotional social interaction among Swedish students, The Internet and Higher Education, 13(4, 197-205 (2010).

Bennett, S., K. Maton y L. Kervin, The "digital natives" debate: A critical review of the evidence, British Journal of Educational Technology, 39(5), 775-786 (2008).

Cabero, J. y V. Marín, Percepciones de los estudiantes universitarios latinoamericanos sobre las redes sociales y el trabajo en grupo, Revista de Universidad y Sociedad del Conocimiento, 10(2), $219-235$ (2013).

Cabra-Torres, F. y G.P. MarCiales-ViVas, Mitos, realidades y preguntas de investigación sobre los 'nativos digitales': una revisión, Revista Universitas Psychologica, 8(2), 323-338 (2009).

Falahaha, S. y D. Rosmala, Study of Social Networking usage in Higher Education Environment, Procedia Social and Behavioral Sciences, 67(10), 156-166 (2012).

Flores, K. y M.C. López, Las motivaciones y los usos sociales y educativos que los estudiantes universitarios hacen del Facebook, Revista de Tecnología y Sociedad, 4(7), 1-10 (2014).

Islas, C. y M. Carranza, Uso de las redes sociales como estrategias de aprendizaje ¿Transformación educativa?, Revista Apertura 3(2), 32-38 (2011).

Kabilan, M.K., N. Ahmad y M.J.Z. Abidin, Facebook: An online environment for learning of English in institutions of higher education?, The Internet and Higher Education, 13(4), 179-187 (2010).

Kimmons, R. y G. Veletsianos, The fragmented educator 2.0: Social networking sites, acceptable identity fragments, and the identity constellation, Computers \& Education, 72, 292-301 (2014).

Llorens, F. y N. Capdeferro, Posibilidades de la plataforma Facebook para el aprendizaje colaborativo en línea, Revista de Universidad y Sociedad del Conocimiento, 8(2), 31-45 (2011).

López, P.L., Población muestra y muestreo, Punto Cero, 69-74 (2004).

McMillan, D.W. y D.M. Chavis, Sense of community: A definition and theory, Journal of Community Psychology, 14(1), 6-23 (1986).

Mendiguren, T., K. Meso y J. Pérez, El uso de las redes sociales como guía de autoaprendizaje en la Facultad de Comunicación de la UPV/EHU, Tejuelo, monográfico, 6, 123-136 (2012).

Molina, R. y S. Briseño, Comunicación y participación en una red virtual de docentes en el área de matemáticas (En línea) 2005.

http://www.ufrgs.br/niee/eventos/RIBIE/2008/pdf/comunicacion_participacion_red.pdf. Acceso: 10 de Marzo del 2015.

Morduchowicz, D., Los adolescentes y las redes sociales, Conexión pediátrica, 5(1), 1-2 (2012).

Moreno, F., La multimedia como herramienta para el aprendizaje autónomo del vocabulario del inglés por parte de los niños, Colombian Applied Linguistics Journal, 13(1), 88-98 (2011). 
Moskaliuk, J., J. Kimmerle y U. Cress, Wiki-supported learning and knowledge building: Effects of incongruity between knowledge and information, Journal of Computer-Assisted Learning, 25(6), 549-561 (2009).

Roblyer, M.D. y otros cuatro autores, Findings on Facebook in higher education: A comparison of college faculty and student uses and perceptions of social networking sites, The Internet and Higher Education, 13(3), 134-140 (2010).

Schmitz, B. y otros tres autores, New developments in the field of self-regulated learning, Journal of Psychology, 215, 153-156 (2007).

Väljataga, T. y S. Fiedler, Supporting students to self-direct intentional learning projects with social media, Educational Technology \& Society, 12(3), 58-69 (2009).

Vidal, M., M.N. Vialart y L. Hernández Redes sociales, Educación Médica Superior, 27(1), 146-157 (2013).

Vivas, M. Moodle: una opción formativa al alcance del profesorado, II Encuentro Internacional de TIC y Pedagogía, Barquisimeto, Venezuela (2006).

Wodzicki, K., E. Schwämmlein y J. Moskaliuk, "Actually, I Wanted to Learn": Study-related knowledge exchange on social networking sites, The Internet and Higher Education, 15(1), 9-14 (2012). 